\title{
Diabetic Foot Profile in Patients under Regular Haemodialysis
}

\author{
Nasreddine Boughallaba ${ }^{1}$ and Habib Ksouri ${ }^{2 *}$ \\ ${ }^{1}$ Centre d'Hémodialyse Nour Echiffa, Avenue Farhat Hached, PB 290, Jerba-Midoun 4116, Tunisia \\ ${ }^{2}$ Service des Laboratoires, Centre National de Greffe de Moelle Osseuse, 2 rue Jebel Lakhdar, Tunis 1006, \\ Tunisia
}

*Corresponding author: Habib Ksouri, Service des Laboratoires, Centre National de Greffe de Moelle Osseuse, 2 rue Jebel Lakhdar, Tunis 1006, Tunisia, Tel: +21671157583

\begin{abstract}
Introduction: Diabetic foot syndrome that affects patients under regular haemodialysis is a complex mechanism mainly based on patient metabolism, neurosensitive abnormalities, and plantar pressure dysfunctions.
\end{abstract}

Patients and methods: Our work is based on a descriptive cross-sectional and multicenter study conducted during 45 days and concerning 43 diabetic patients among a total of 193 patients undergoing regular haemodialysis in three haemodialysis centers in Tunisia.

Data collection relied on patients clinical records, all data were collected anonymously and we use 3 sheets respectively for: Data collection, clinical examination and neuropathic pain evaluation.

Some biological parameters which are associated with chronic kidney disease (CKD) were studied, as parathyroid hormone (PTHi), phosphatemia, hemoglobin $(\mathrm{Hb})$, glycosylated haemoglobin ( $\mathrm{HbA} 1 \mathrm{c})$.

Passive articular mobility was explored by a goniometer which is a tool for measuring joint amplitudes. Lower limbs arteriopathy exploration as: Ankle-brachial pressure index (ABPI) was performed using a pocket continuous wave doppler ( $8 \mathrm{Mhz}$ ) and a sphygmomanometer with a cuff width of at least the third of limb circumference. Protective skin sensitivity was explored by Semmes-Weinstein monofilamant $10 \mathrm{~g}$ (SWM), and tactile skin sensitivity was explored by a cotton strand (wadding).

As samples were small, we used Fisher's exact test for paired series for the statistical analysis of the relationship between certain parameters and diabetic foot.

Results: The majority of the patients have type 2 diabetes. All had diabetic nephropathy and several comorbidities, $57 \%$ secondary hyperparathyroidism, $35 \%$ had a normalized proteins catabolic rate, and $44.1 \%$ had hypoalbuminemia. Fifty one percent of the patients have feet deformities as, toes claws $(44.1 \%)$, hallux valgus $(25.6 \%)$ and a history of ulceration or amputation (34.8\%). Thirteen patients had diabetic neuropathy and $53.48 \%$ had sensory neuropathy. Neuropathic pain was found in $18.6 \%$ of patients. Regarding factors related to haemodialysis, the impact of "nutritional, inflammatory, anemic and disorders of the metabolism of calcium and of phosphorus" factors was clear. Diabetes inherent factors such as, duration of evolution, balance, and the tendency to obesity represent others complicating risk factors for our patients. Also, ABPI, which constitutes another risk factor, was greatly increased. Similarly, sensory and autonomic neuropathies were strongly present, as well as bone deformities.

Conclusion: Obesity, malnutrition and comorbidities, as poorly balanced diabetes, anemia, disorders of the metabolism of calcium and phosphorus associated with a chronic inflammatory state, constitute risk factors that promote the occurrence of the diabetic foot complications. Presence of sensory and autonomic neuropathy, bone deformities and vascular calcification also contribute to the development of such pathology.

\section{Keyword}

Diabetes complications, Diabetic foot, Haemodialysis, Neuropathies

\section{List of Abbreviations}

ABPI: Ankle-Brachial Pressure Index; BMI: Body Mass Index; CKD: Chronic Kidney Disease; CRP: C Reactive Protein; CVD: Cardio-Vascular Disease; DP: Dorsalis Pedis; HbA1c: Glycosylated Haemoglobin; npcr: Normalized Protein Catabolic Rate; PAD: Peripheral Arterial Disease; PTA: Posterior Tibial Artery; PTHi: Parathyroid Hormone; SWM: Semmes-Weinstein Monofilament; UKPD: United Kingdom Prospective Diabetes Study Cohort; URR: Urea Reduction Ratio 


\section{Introduction}

Chronic kidney disease (CKD) treated with regular haemodialysis is increasing dramatically worldwide, and diabetes which is responsible for $20 \%$ to $45 \%$ of dialysis treatment represents its most common cause [1]. Diabetic patients on dialysis have a high cardiovascular risk [1] because renal failure is an independent risk factor for arthritis development.

The diabetic foot syndrome which characterizes the course of survival of patients treated by regular haemodialysis lies upon complex mechanisms, among which "accelerated atherosclerosis", metabolic disorders including phosphorus and calcium balance, malnutrition, and inflammation. Anemia plays a significant role when it is present, and hypertension (High Blood Pressure) greatly aggravates the prognosis of vascular disease [2] The pathogenesis also includes other mechanisms, such as: the development of a diabetic polyneuropathy, as well as a diabetic macro and microangiopathy associated with immunological incompetence as part of a chronic metabolic impairment. Inflammation gains increasing interest as a contributing factor to the increased CVD (Cardio-Vascular Disease) risk in patients with CKD.

Serum biomarkers of inflammation, such as C-reactive protein (CRP) and IL-6, are associated with increased CVD risk, not only in the general population, but also in patients with CKD $[3,4]$. In addition to systemic inflammation, local inflammatory activity of the arterial wall is also implicated in such phenomenon [5].

Bernelot Moens, et al. [6] found an increased arterial wall inflammation that correlates directly to measures of kidney function in patients with CKD, without known atherosclerotic or inflammatory disease, and with few traditional risk factors and comorbidity.

Medial calcification is also a prominent pathology in patients with CKD and progresses rapidly in patients on dialysis $[7,8]$. This calcification is associated with increased vascular stiffening and cardiac workload, poor coronary perfusion, and sudden cardiac death and is probably responsible for the high cardiovascular mortality observed in CKD patients. The prevalence of such complications is 2.5 times higher in patients on dialysis than in non-dialysis one [4].

CKD invariably increases the risk of ulceration and amputation for diabetic foot, so progression from diabetic nephropathy to end-stage chronic renal failure is associated with a significant increase in morbidity and mortality caused by ulceration and amputation [9]

Foot ulceration is a common complication that affects up to $25 \%$ of diabetic patients during their lifetime. Moreover, the very high prevalence of foot ulceration in diabetic patients on dialysis is probably a consequence of end-stage renal disease [10].

Remind that patients with a history of foot ulcer have a 34-fold increased risk of ulcer recurrence [3] and that the in-hospital and 30-day mortality after amputation in people with diabetes is higher than in people with coronary artery bypass graft surgery, breast cancer, or stroke [11-13]. Direct costs of diabetic foot ulceration care for health system due to are high; these costs are doubled when foot ulceration leads to amputation [14].

During this work, we proposed to study the clinical profile of diabetic foot in CKD Tunisian patients undergoing regular haemodialysis. Even if we worked on a small sample; we tried to demonstrate if our patients' particularities based on risk and protective factors to develop diabetic foot complications are in agreement with what reported in literature.

So we evaluated the impact of some factors on diabetic foot complications as, age $[1,15,16]$, co-morbidities $[1,9,17,18]$, socio-economic level [19].

The effect of factors related respectively to haemodialysis as nutritional [20-24] and inflammatory state $[3-5,10,20,23,24]$, anemia $[16,17,25]$, calcium and phosphorus metabolism disorders $[16,21]$ and to diabetes as, duration of evolution $[16,26,27]$, balance $[16,17,25]$, tendency to obesity $[1,16,27]$ on the development of foot complications, were also evaluated. Always, by comparing with literature data, we have described types of diabetic foot lesions $[15,25,28]$, type of diabetic neuropathies [29] and calculate ABPI to explorate lower limbs arteriopathy of our patients $[28,30]$.

\section{Patients and Methods}

\section{Type and study setting}

Our work is based on a descriptive cross-sectional and multicenter study conducted from 04/15/2017 to $05 / 30 / 2017$ and concerning 43 diabetic patients (22.27\%) among a total of 193 patients undergoing regular haemodialysis in three haemodialysis centers (the el Khadra haemodialysis service (polyclinique CNSS), the dialysis service at the Ben Arous Regional Hospital and the Echiffa dialysis clinic at Jerba).

\section{Methodology}

Data collection relied on patients clinical records, all data were collected anonymously and we use 3 sheets respectively for: data collection (Annex 1), clinical examination (Annex 2) and neuropathic pain evaluation (DN4 Questionnaire).

Passive articular mobility was explored by a goniometer which is a tool for measuring joint amplitudes. Roaas, et al. [29] give values of $20^{\circ}$ in dorsal flexion and $50^{\circ}$ to $40^{\circ}$ in plantar flexion. We measure the amplitudes in dorsal and plantar flexion of the talo-crural joint which associates the movements of the other joints located more distally. The mobility standards values are: Active: "Dorsal flexion/Plantar flexion" $=\left(20^{\circ} / 0^{\circ} / 40^{\circ}\right)$ Passive: "Dorsal flexion/Plantar flexion" $=\left(30^{\circ} / 0^{\circ} / 50^{\circ}\right)$. 
Measurements of the dorsal flexion of the talo-crural joint are performed in knee flexion; patient's is in strict dorsal decubitus free from heels support. Measurements are made as following, the goniometer is positioned along joint axis (the tip of the lateral malleole), the fixed branch targets the head of the fibula and the moving branch targets the head of the $5^{\text {th }}$ metatarsal. The neutral reference position corresponds to the anatomical position, the sole of the foot which forms an angle of $90^{\circ}$ with the leg. In this position, there is an angle of about $115^{\circ}$ which must be deduced.

Lower limbs arteriopathy exploration as: Ankle-brachial pressure index (ABPI) was performed using a pocket continuous wave Doppler ( $8 \mathrm{MHz}$ ) and a sphygmomanometer with a cuff width of at least the third of limb circumference. ABPI is an invaluable tool for assessing the severity of peripheral arterial disease (PAD). The Society for Vascular Technology [31] and the American Heart Association have produced guidelines for the measurement of ABPI [32]. Patient is laying supine, ankles supported and knees straight, with their head supported. Before beginning the measurements, patients should be in rest for approximately $15 \mathrm{~min}$, because physical exercise can distort the value of ABPI. The Doppler is then used to measure the blood pressure. Both the right and left brachial arteries pressure need also to be measured to determine the denominator of the equation and if there's a difference, the higher value obtained should be used. The examiner subsequently measures the blood pressure at the level of the ankles, placing a Doppler probe over the dorsalis pedis (DP) and then the posterior tibial artery (PTA). The values obtained are then used to calculate the ABPI using the formula: ABPI $=$ Highest PTA or DP systolic pressure in examined limb/ Highest brachial Systolic pressure. ABPI range: [> 1.3: Consider presence of calcification; 1.0-1.3: Likely normal; 0.9-1.0 borderline PAD; < 0.9: Claudication].

Protective skin sensitivity was explored by Semmes-Weinstein monofilament 10g (SWM), and tactile skin sensitivity was explored by a cotton strand (wadding). The SWM testing is a clinical test that measures the response to a touching sensation of the monofilaments using a numerical quantity, and it's used for the detection of patients at risk of neuropathic ulceration, and in the evaluation of peripheral nerve injuries and compression syndromes [33]. Practical examination of the surface sensitivity by the monofilament test $10 \mathrm{~g}$ is described in the international recommendations on the prevention of diabetic foot [34]. The examination is carried out calmly. First apply the monofilament on the patient's hands (or on the elbow or forehead) so that he knows what he must be feeling. The patient should not see whether or not the examiner is applying the filament, or where apply it. The three sites to be tested on each foot are: The plantar side of the head of the first metatarsal, head of the fifth metatarsal and the pulp of hallux. Apply the filament perpendicular to the surface of the skin with enough force to cause its' bulge. The total duration of the test including "approaching the filament, skin contact and removal", should last approximately 2 seconds. During the test the patient must be encouraged. Certain rules must be applied: "Apply the filament on the periphery and not on the site of the ulcer; do not apply it on a callus, scar or necrotic tissue (Do not do it slide on the skin or make repeated contacts in the area explored); press the filament on the skin and ask the patient if he feels the pressure applied (yes/ no) and where he feels it (right/left foot); repeat the application twice at the same site by inserting a "dummy test" at the during which the filament is not applied (in total: 3 questions per site). Results interpretation: The feeling of protection is preserved at each site if the patient responds correctly to two of the three applications. It is absent if two of the three answers are wrong: the patient is then considered at risk of ulceration.

\section{Statistical analysis}

In order to ensure the maximum homogeneity of collection and input, all data was collected and entered by one person.

As samples were small ( $<5$ in some cases) we used Fisher's exact test for paired series for the statistical analysis of the relationship between certain parameters and diabetic foot complications [ $(\alpha=5 \%)$; 'Confidence Interval' $\mathrm{Cl}=95 \%]$.

SPSS software (Statistical Product and Services Solutions, version 18.0, SPSS Inc, Chicago, IL, USA) was used for statistical analyses.

\section{Results}

\section{Patients' characteristics}

Among all 43 diabetic undergoing regular haemodialysis patients, only fifteen had serious podiatry problems as amputations or ulcers.

Mean age of patients was $59.6 \pm 11.9$ years "(extremes (32-83)" 76\% among them were male. All of our patients live in urban areas, $76.75 \%$ are of a good socioeconomic level and $48.8 \%$ are illiterate or of primary school level.

The majority of our patients have type 2 diabetes (76.75\%) and $23.25 \%$ have type I diabetes. For $81.3 \%$ of them, the onset of diabetes was beyond ten years.

There wasn't any significant difference between patients under and over 10 years diabetes evolution concerning diabetic foot complications $(p=0.24)$ (Table 1 ) and all of them had diabetic nephropathy.

All patients have simultaneously several co-morbidities, $53.5 \%$ severe retinopathy requiring laser treatment, $55.8 \%$ coronary artery disease, $46.5 \%$ hypertension, and $72 \%$ dyslipidemia. Twenty three patients (53.4\%) were overweight and $27.9 \%$ were obese. 
Table 1: Distribution of patients by duration of diabetes.

\begin{tabular}{|l|l|l|l|}
\hline Duration of diabetes & Patients with amputation or ulcer & Unaffected patients & Total Patients \\
\hline $\mathbf{> 1 0}$ years & 9 & 21 & 30 \\
\hline $\mathbf{1 0}$ years & 6 & 7 & 13 \\
\hline Total Patients & 15 & 28 & 43 \\
\hline
\end{tabular}

$\mathrm{p}=0.24$.

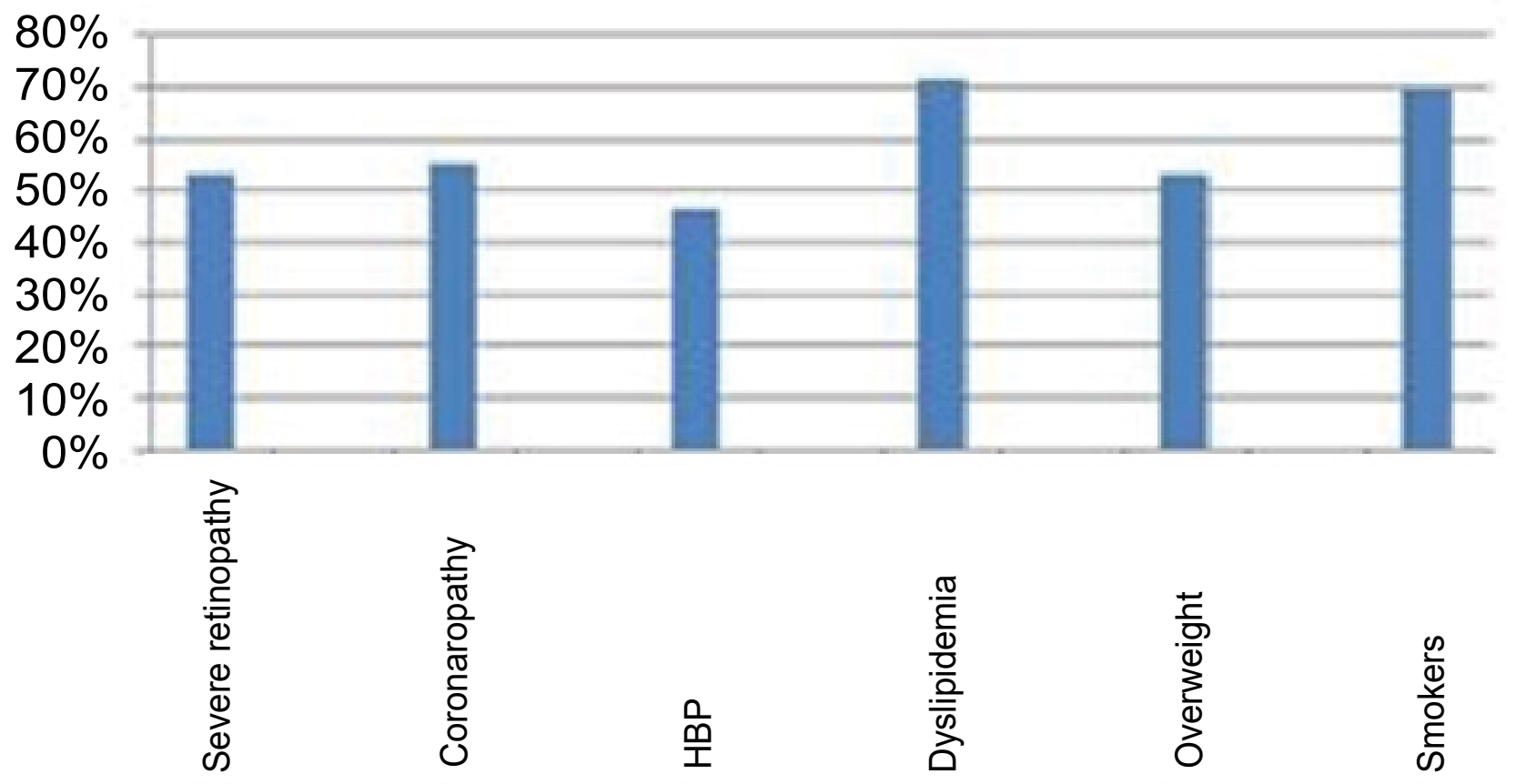

Figure 1: Percentage of comorbidities.

HBP: High Blood Pressure.

There was no significant difference $(p=0.5)$ between patients with diabetic foot complications and those without, based on BMI category.

Seventy percent of patients are smokers $(60 \%$ active, $40 \%$ former), and also there wasn't any significant difference concerning diabetic foot complications between smokers and no smokers $(p=0.13)$.

Three patients have had respectively, one an ischemic stroke, the second a hemorrhagic stroke and arrhythmia for the third one (Figure 1).

The majority of patients, $90.7 \%$ have been undergoing regular haemodialysis for less than ten years, $20.5 \%$ among them for less than one year, 9.3\% have been undergoing regular haemodialysis for more than ten years.

Duration average in regular haemodialysis was of 56 months.

All patients receive twelve hours of dialysis, three sessions per week.

The average urea reduction ratio (URR) was $72 \%$ (50\% to $79 \%)$.

More than ninety percent (90.7\%) of patients have a native arteriovenous fistula and $9.3 \%$ a central venous catheter.

Eighty percent of fistulas are proximal (humeral) and $20 \%$ distal (radial).

Six patients $(13.9 \%)$ received three or more arteriovenous fistulas, $25.5 \%$ benefited from two fistulas and four patients (9.3\%) benefited from central venous catheters including a permanent tunneled catheter.

\section{Biological parameters}

The mean value of glycosylated haemoglobin ( $\mathrm{HbA1c}$ ) in our patients is $8.37 \% \pm 2.35 \%(5.7 \%-13.5 \%)$ [(68 $\pm 2 \mathrm{mmol} / \mathrm{mol})(39-124 \mathrm{mmol} / \mathrm{mol})]$.

Three-quarters of the patients $(74.4 \%)$ are anaemic with haemoglobin levels below $11 \mathrm{~g} / \mathrm{dl}$. Fifty-seven percent of our patients have secondary hyperparathyroidism, $14 \%$ have hypoparathyroidism with a Parathyroid hormone (PTHi) levels $<150 \mathrm{pg} / \mathrm{ml}$. Hyperphosphoremia is present in $46 \%$ of our patients.

The normalized protein catabolic rate (npcr) represents the rate of normalized protein catabolism, which evaluates the amount of protein taken by a patient between two dialyses. An npcr below 1 is defined as a criterion of undernutrition. 


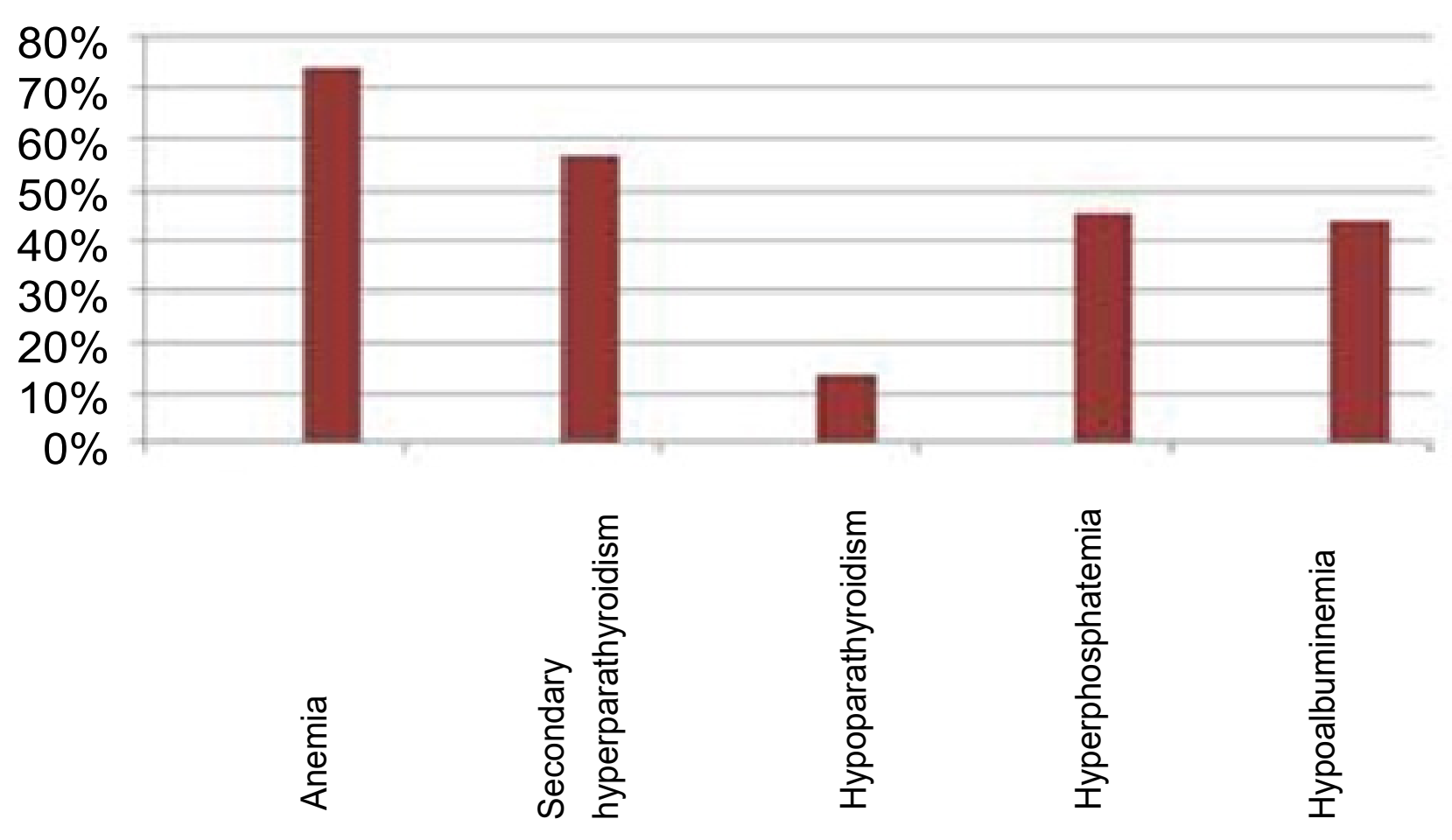

Figure 2: Percentages of biological abnormalities.

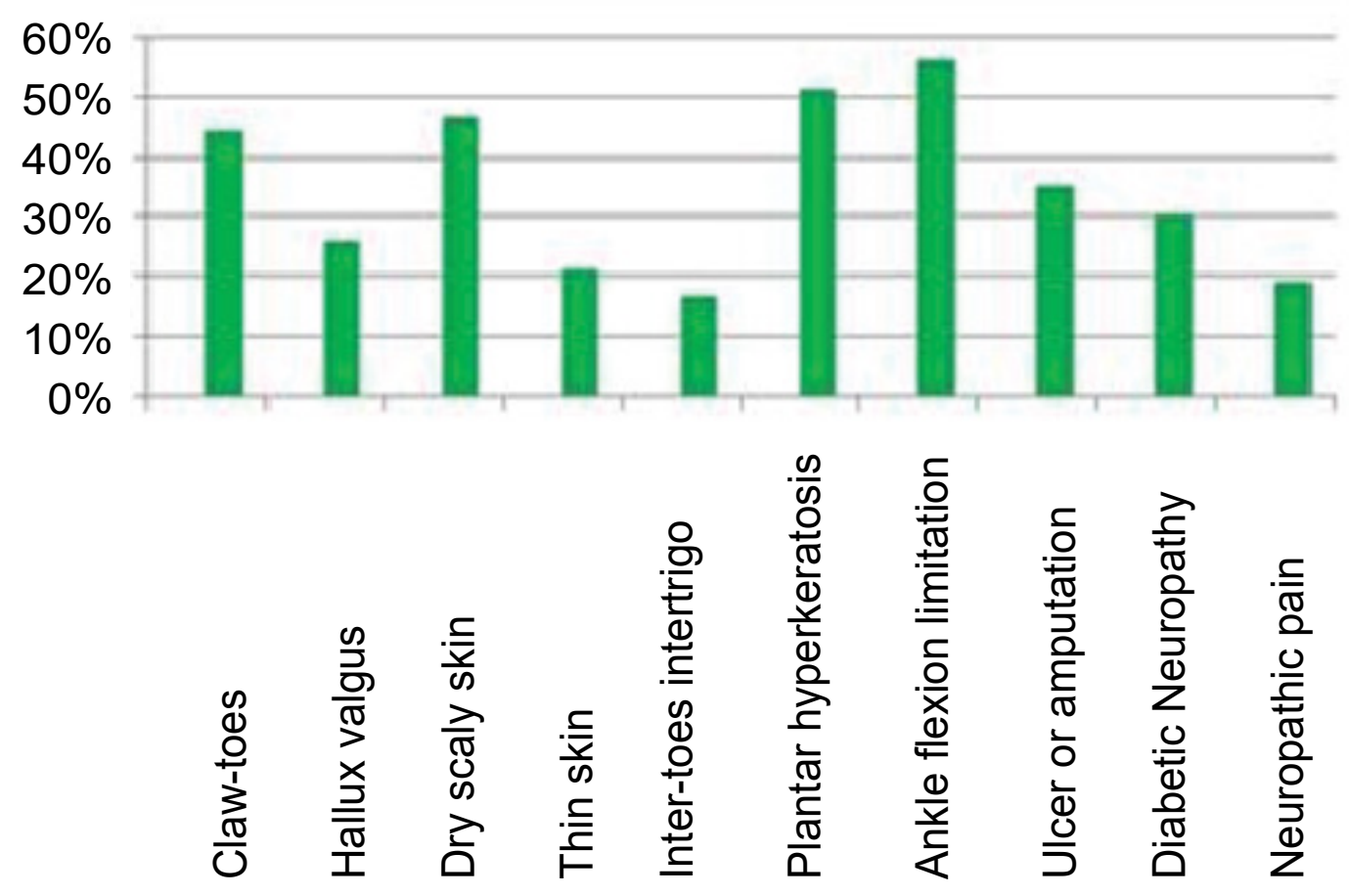

Figure 3: Percentages of the main clinical podiatry data.

Thirty-five percent of our patients have low blood pressure, and $44.1 \%$ have hypoalbuminaemia, mean 39 $\mathrm{g} / \mathrm{l} \pm 3.5 \mathrm{~g} / \mathrm{l}(33 \mathrm{~g} / \mathrm{l}-48 \mathrm{~g} / \mathrm{l})$.

Seventeen patients (39.5\%) have $\mathrm{C}$ reactive protein (CRP) level above $10 \mathrm{mg} / \mathrm{l}$, mean: $11.76 \mathrm{mg} / \mathrm{I} \pm 9.63$ $\mathrm{mg} / \mathrm{l}(1 \mathrm{mg} / \mathrm{l}-36 \mathrm{mg} / \mathrm{l}$ ) (Figure 2).

Distribution according to the clinical examination

Deformation or bone prominence: Twenty two pa- tients (51.16\%) have feet deformities, among them $44 \%$ have claw-toes, eight of which are bilateral and nine of claw-toes are little or none at all reducible and $25.6 \%$ of patients have hallux valgus, three of which are bilateral. In $23 \%$ of patients hallux valgus is associated with clawtoes (Figure 3).

Skin condition, joint mobility and history of ulcer or amputation: Only one patient had infected gangrene of the fifth toe, $46.5 \%$ had dry, sometimes scaly skin, 
Table 2: Distribution of patients according to sensitive protective neuropathy.

\begin{tabular}{|l|l|l|l|}
\hline Sensitive protective neuropathy & Patients with amputation or ulcer & Unaffected patients & Total Patients \\
\hline Present & 8 & 22 & 30 \\
\hline Absent & 7 & 6 & 13 \\
\hline Total Patients & 15 & 28 & 43 \\
\hline
\end{tabular}

$\mathrm{p}=0.08$.

Table 3: Distribution of patients according to neuropathic pain.

\begin{tabular}{|l|l|l|l|}
\hline Neuropathic pain & Patients with amputation or ulcer & Unaffected patients & Total Patients \\
\hline Present & 3 & 5 & 8 \\
\hline Absent & 12 & 23 & 35 \\
\hline Total Patients & 15 & 28 & 43 \\
\hline
\end{tabular}

$p=0.5$.

Table 4: Distribution of patients according to ABPI.

\begin{tabular}{|l|l|l|l|}
\hline ABPI & Patients with amputation or ulcer & Unaffected patients & Total Patients \\
\hline Pathological & 12 & 17 & 29 \\
\hline Normal & 3 & 11 & 14 \\
\hline Total Patients & 15 & 18 & 43 \\
\hline
\end{tabular}

ABPI: Ankle-brachial pressure index; $p=0.17$.

and $20.9 \%$ had thin skin. Twenty-two patients $(51.16 \%)$ had plantar hyperkeratosis in the metatarsal head, with in two cases an association with hyperkeratosis of the heel.

Twenty-four patients (56\%) had a limitation of ankle flexion. Fifteen $(34.88 \%)$ of our patients have a history of ulcer or amputation, in fact, respectively six patients had a minor, and four a major amputation, and five have sequelae of foot ulcer. One patient was in a plaster boot following a fracture of the fifth metatarsal.

Neuropathies and neuropathic pain: Thirteen of our patients (30\%) had diabetic neuropathy with a loss of protective sensitivity, and 23 patients $(53.48 \%)$ had a sensory neuropathy without attaining protection sensitivity and with less than four symptoms.

Neuropathic pain was found in $18.6 \%$ of our patients. As well for sensitive protective neuropathy (Table 2) and neuropathic pain (Table 3) there wasn't any significant difference between patients with or without diabetic foot complications.

Index of ankle/arm systolic pressure: Pedal and posterior tibial pulses were present in all our patients, but were difficult to detect in $25.5 \%$ of cases. Thirty patients (69.7\%) have an $\mathrm{ABPI}>1.3 ; 7 \%$ an $\mathrm{ABPI}<0.9$ and $21 \%$ an $A B P I$ within the normal range (Table 4).

Distribution according to the footwear: Only one of our patients has suitable footwear.

\section{Discussion}

The average age of our population is 59.6 years, which is comparable to that found by Kabbali, et al. [1] in Morocco ( $59 \pm 13.2$ years), in his study including 2066 patients undergoing regular haemodialysis. Similarly, Benabdallah, et al. [15] find an average age of 52 years + - 5.6 years.

On the other hand, Elouazzani, et al. [16] in a study of 35 French diabetic undergoing regular haemodialysis patients, found an average age of 74.5 years. This age difference could be explained by a higher expectation of age among the French population suffering from such pathology, probably thanks to the more adapted care of this population.

Nather, et al. [19] found statistically significant differences in socio-economic factors between diabetic patients with diabetic foot problems and those without such problems. Low monthly income and level of education (no high school) are among the factors that were significantly higher in patients suffering from diabetic foot complications.

For our patients, the diagnosis of the type of nephropathy remains rather a clinical and biological presumption since no patients had a renal tissue biopsy. Diabetic nephropathy is a leading cause of end-stage renal failure.

The duration of evolution of diabetes is generally considered as a factor favoring the occurrence of ulceration [27]. The fact that more than two-thirds of our patients (76.6\%) are suffering from type 2 diabetes with over 10 years evolution, is consistent with the results of Vilar, et al. [26] who found that among patients suffering from diabetes in their cohort $84 \%$ was suffering from type 2 diabetes. Similarly, Elouazzani, et al. [16] found that in their series of 35 diabetic patients undergoing haemodialysis, all patients had type 2 diabetes with a 
mean duration of $19.7 \pm 13.3$ years. For our patients, we found no statistically significant difference between amputees and non-amputees in relation to the duration of diabetes evolution (Table 1).

The high frequency of co-morbidities in our diabetic patients undergoing regular haemodialysis (Figure 1), is consistent with the frequencies reported by Kabbali, et al. [1] who found $22.6 \%$ patients with cardiovascular co-morbidities (whose $62.4 \%$ were ischemic heart disease, $27.7 \%$ heart failure and $64 \%$ high blood pressure).

Stamm, et al. [17] report that $54 \%$ of such patients developed at least one macrovascular complication of diabetes, 64\% had probable diabetic retinopathy and $82 \%$ had hypertensive disease.

We found that $53.4 \%$ of our patients are in overweight and $27.9 \%$ are obese. Such results were reported by Kabbali, et al. [1] who found that $21 \%$ of their patients are obese and $78 \%$ are in overweight. The analysis of the clinical data of Elouazani, et al. [16] shows a statistically significant difference in Body Mass Index (BMI) for patients undergoing regular haemodialysis with a tendency to obesity in the diabetics group. In his study of the relationship between the degree of kidney failure and diabetic foot complications, Wolf, et al. [27] found an average BMI of $26.6 \%$ in type 1 diabetic patients and $30 \%$ in type 2 diabetic patients.

The aggravating role of smoking, which is a classic feature in the setting up and progression of atherosclerosis, is of great importance for lower extremity arterial disease [18]. In a multi-varied analysis, active smoking is associated with a relative risk of occurrence of arterial disease of the lower limbs almost triple (OR $=2.90, \mathrm{Cl}$ $=1.46-5.73)$, far ahead of the age, the $\mathrm{HbA} 1 \mathrm{c}$, systolic blood pressure or HDL cholesterol.

For our patients, there was no statistical significant difference between amputees and unaffected ones concerning BMI and smoking.

A mean duration of 56 months of regular haemodialysis, was noted for our patients, which was close to means reported by Kabbali, et al. [1] (39.3 \pm 67 months) and Benabdellah, et al. [4] (69.3 +/- 11 months).

Dialysis Outcomes and Practical Patterns Study (DOPPS) [20] show a positive correlation between haemodialysis dose and survival, both in the United States and Europe.

Only $69.7 \%$ of our patients have an URR $>65 \%$, which does not meet international recommendations.

Regarding seat and blood access, our results are similar to those of Kabbali, et al. [1] and Al-Thani, et al. [21].

Indeed, Kabbali, et al. found that 435 of their patients are dialyzed on a distal arterio-venous fistula (AVF) in $74.8 \%$, and proximal in $22.5 \%$, with 12 patients dialyzed on a permanent catheter. In their observational study on the involvement in haemodialysis of the foot ulcer, Al-Thani, et al. [21] found that there was no statistically significant difference in the number of access to blood between patients with or without foot ulcers.

Patients in both groups had more than three accesses to blood respectively in $47 \%$ of cases for foot ulcers and $60 \%$ for those without foot ulcers. Diabetes appears as a factor altering the survival of AVF.

As reported in the literature $[16,17,25]$, our patients have several types of biological abnormalities (Figure 2).

$\mathrm{HbA1c}$ remains the reference marker for diabetes equilibrium, although it almost always underestimates the reality of blood glucose equilibrium in patients under regular haemodialysis. In our series, the mean of $\mathrm{HbA} 1 \mathrm{c}$ of $8.3 \%(68 \mathrm{mmol} / \mathrm{mol})$ is at the high limit of results reported by Elouazzani, et al. [16], but a little higher than Stamm, et al. [17] which are respectively 6.9 +/- 1.4\% (37 mmol/mol to $67 \mathrm{mmol} / \mathrm{mol}$ ) and 6.93\% (52 $\mathrm{mmol} / \mathrm{mol})$.

The normal value of $\mathrm{HbA} 1 \mathrm{c}$ in patients under regular haemodialysis is [(6.5\% to $8.5 \%)$; $(48 \mathrm{mmol} / \mathrm{mol}$ to 69 $\mathrm{mmol} / \mathrm{mol})]$.

The average $\mathrm{Hb}$ value in our patients was $9.69 \mathrm{~g} /$ $\mathrm{dl}$, which is below the values found by Stamm, et al. [17], Ndip, et al. [25] and Elouazzani, et al. [16] who are around $11.5 \mathrm{~g} / \mathrm{dl}$. This fact would probably be due to malnutrition and parathyroid disorders in our patients.

We found an average PTHi of $682.3 \mathrm{pg} / \mathrm{ml}$, which corresponds to a secondary hyperparathyroid condition in our patients.

However, Elouazzani, et al. [16] found a PTHi of $300.17 \mathrm{pg} / \mathrm{ml}$, while AlThani, et al. [21] found a value of $423.4 \mathrm{pg} / \mathrm{ml}$ for patients with no foot ulcers, and 526 $\mathrm{pg} / \mathrm{ml}$ for those with foot ulcers.

The mean phosphorus level in our study is 1.89 $\mathrm{mmol} / \mathrm{l}$ corresponding to hyperphosphoremia, which is consistent with Elouazzani, et al. [16] and AlThani, et al. [21] which found respectively a phosphorus level of $1.63 \mathrm{mmol} / \mathrm{l}$ and $1.62 \mathrm{mmol} / \mathrm{l}$ corresponding also to a hyperphosphoremia.

Regarding the prevalence of undernutrition, our results are consistent with those of Hassani, et al. [22] who found a prevalence of undernutrition of $30 \%$ according to protein intake, recalling that $35 \%$ of our patients have an insufficient protein intake. Elouazzani, et al. [23] found an average albumin of $38.4 \pm 4.3 \mathrm{~g} / \mathrm{l}$ which approximates our value of $39.05 \mathrm{~g} / \mathrm{l}$.

In patients under regular haemodialysis, the prevalence of chronic inflammation, with no infectious or neoplastic identifiable cause, is important. With regard to the average $C$ reactive protein (CRP) of $11.76 \mathrm{mg} / \mathrm{I}$ found in our patients, the values agree with those of 
Elouazzani, et al. [23], which found an average CRP of $12.3 \pm 7.1 \mathrm{mg} / \mathrm{l}$ (Normal value $=5$ to $10 \mathrm{mg} / \mathrm{l})$.

On the other hand, this state of malnutrition in diabetic patients under regular haemodialysis can be explained by the chronic inflammatory state which associates various complications, in particular the increase of the anemia secondary to the chronic renal failure and the deterioration of the nutritional state; inadequate intake associated with increased needs; poorer dialysis and the anorexia state due to uremia effect [24].

Our patients suffer from several types of diabetic foot lesions (Figure 3). Our results are consistent with those of Ndip, et al. [25], who found a prevalence of $21 \%$ foot ulcer, $20 \%$ deformity, $15 \%$ amputation. Similarly, Benabdellah, et al. [15] found a frequency of $58.3 \%$ of static disorders, $16.6 \%$ of trophic disorders and $8 \%$ of amputations. Otte, et al. [28] also found a frequency of $15.8 \%$ ulcer history, $2.7 \%$ lower limb amputation and $13.9 \%$ foot deformity.

With respect to diabetic neuropathy, our results are similar to those of Otte, et al. [28] who reported $26.9 \%$ of diabetic peripheral neuropathy in the group of diabetic haemodialysis patients, and who used the same diagnostic means as those we used.

On the other hand, Ndip, et al. [25] found a high prevalence of neuropathy of $79 \%$, such difference is probably due to the fact that Ndip [25] used the neurothesiometer, which is a more sensitive examination tool. There was no significant difference in our work concerning this neuropathy between amputees and unaffected patients (Table 2 ).

Thirty-five percent of our patients have either an amputation or a history of ulcer, which is consistent with Otte, et al. [28] who found in the group of diabetic under regular haemodialysis patients a percentage of $36.8 \%$ of arteriopathy based on a clinical definition.

According to Richard, et al. [30], ABPI does not represent a potential risk factor for ulceration, which is apparent in our patients, since we found no statistically significant differences in ABPI between amputees and unaffected patients (Table 4).

Only one of our patients has suitable footwear, which reflects the blatant lack of care for these patients at high podiatric risk. Our result is consistent with that of Benabdellah, et al. [15] who found a very small percentage $(8 \%)$ of patients wearing adapted shoes.

Our work provides a general overview on the complications of diabetic feet in Tunisian patients under regular haemodialysis, and the main causes that lead to such complications. By referring to the data of the literature, the majority of the socio-economic, clinical and biological parameters, place our patients in the category of patients followed in developing countries. The high frequency of co-morbidities in our diabetic patients under- going regular haemodialysis, the existence of a chronic inflammatory state, although of low level, are all factors which favor diabetic foot complications. Irregular monitoring of patients and their indiscipline regarding the monitoring of hygienic-dietary rules, either through ignorance or lack of resources, are all aggravating factors.

The size of the sample analyzed and the lack of more convincing tools of exploration represent a significant bias in our work. A larger study, recruiting a substantial number of patients and more detailed analyzes of the different clinical and biological parameters would be more adequate. In addition, better monitoring and better education of patients at risk would be a better asset to avoid deleterious complications of the foot in these patients.

\section{Conclusion}

The tendency to obesity in diabetic patients often malnourished, associated with the presence of many comorbidities, diabetes often poorly balanced, chronic inflammatory state, anemia and disorders of the metabolism of calcium and phosphorus, are all factors that promote the occurrence of the diabetic foot complications. Similarly, the presence of sensory neuropathy, autonomic neuropathy, bone deformities and vascular calcification also contribute to the development of such pathology.

\section{Consenting of the Study Subjects}

Biological testing and some clinical examinations are part of the patients' routine exam, nonetheless, patients were informed about the purpose of this work and they give all their consenting.

\section{Conflict of Interest}

The authors declare that they have no conflict of interest.

\section{Competing Interests}

The authors declare no competing interest.

\section{Acknowledgements}

Thanks to: Dr. Asma Ben Nour, Director of Nour Echiffa Haemodialysis Center, Jerba, Tunisia; Dr. Sonia Bebchia, Director of Haemodialysis Center, Ben Arous Regional Hospital; Dr. Raoudha BelHaj, Director of Haemodialysis Center, Cité El Khadra Clinic, Tunisia.

\section{References}

1. Kabbali N, Mikou S, El Pardiya NT, El Bardai G, Arrayhani M, et al. (2014) Profile of diabetic in chronic haemodialysis: A multicenter study in Morocco. Pan Afr Med J 17: 125.

2. de Belsunce M, Barnay C, Kessali V (1981) Cardiovascular complication of renal dialysis. 2. Changes in the cardiovascular system in patients treated with periodic haemodialysis. Rev Med Interne 2: 159-166. 
3. Zebrack JS, Anderson JL, Beddhu S, Horne BD, Bair TL, et al. (2003) Do associations with C-reactive protein and extent of coronary artery disease account for the increased cardiovascular risk of renal insufficiency? J Am Coll Cardiol 42: 57-63.

4. Knight EL, Rimm EB, Pai JK, Rexrode KM, Cannuscio CC, et al. (2004) Kidney dysfunction, inflammation, and coronary events: A prospective study. J Am Soc Nephrol 15: 1897-1903.

5. Rudd JHF, Myers KS, Bansilal S, Machac J, Rafique A, et al. (2007) (18)Fluorodeoxyglucose positron emission tomography imaging of atherosclerotic plaque inflammation is highly reproducible: Implications for atherosclerosis therapy trials. J Am Coll Cardiol 50: 892-896.

6. Bernelot Moens SJ, Verweij SL, van der Valk FM, van Capelleveen JC, Kroon J, et al. (2002) Arterial and cellular inflammation in patients with CKD. J Am Soc Nephrol 28: 1278-1285.

7. Goodman WG (2002) Vascular calcification in end-stage renal disease. J Nephrol 15: S82-S85.

8. Goodman WG, Goldin J, Kuizon BD, Yoon C, Gales B, et al. (2000) Coronary-artery calcification in young adults with endstage renal disease who are undergoing dialysis. $\mathrm{N}$ Engl J Med 342: 1478-1483.

9. Margolis DJ, Hofstad O, Feldman HI (2008) Association between renal failure and foot ulcer or lower-extremity amputation in patients with diabetes. Diabetes Care 31: 13311336.

10. Kerr M, Barron E, Chadwick P, Evans T, Kong WM, et al. (2019) The cost of diabetic foot ulcers and amputations to the National Health Service in England. Diabetic Med 36: 995-1002.

11. O'Hare AM, Feinglass J, Reiber GE, Rodriguez RA, Daley $J$, et al. (2004) Postoperative mortality after nontraumatic lower extremity amputation in patients with renal insufficiency. J Am Soc Nephrol 15: 427-434.

12. Kunadian B, Dunning J, Millner RW (2007) Modifiable risk factors remain significant causes of medium term mortality after first time Coronary artery bypass grafting. J Cardiothorac Surg 2: 51.

13. Halkos ME, Puskas JD, Lattouf OM, Kilgo P, Kerendi F, et al. (2008) Elevated preoperative hemoglobin A1c level is predictive of adverse events after coronary artery bypass surgery. J Thorac Cardiovasc Surg 136: 631-640.

14. NHS England (2019) Programme budgeting.

15. Benabdellah N, El Harraqui R, Karimi I, Bentata Y, Haddiya I (2012) What about podiatric condition in chronic haemodialysis patients with diabetes? Nephrol Ther 8: 330.

16. Elouazzani H, Sirajedine K, Aladib M (2012) Diabetes dialysis: Prevalence and comorbidities associated with chronic haemodialysis population. Nephrol Ther 8: 335

17. Stamm C, Burnier M, Zanchi A (2011) Diabetes and end stage renal disease. Eight year progression in the Canton de Vaud, Switzerland. Rev Med Suisse 7: 495-499.

18. Nativel M, Potier L, Alexandre L, Baillet-Blanco L, Ducasse $E$, et al. (2018) Lower extremity arterial disease in patients with diabetes: A contemporary narrative review. Cardiovasc Diabetol 17: 138.

19. Nather A, Siok Bee C, Keng Lin W, Qi Odelia KS, Yiong Huak C, et al. (2010) Socioeconomic profile of diabetic patients with and without foot problems. Diabet Foot Ankle 1: 10.
20. Combe C (2010) Dialysis dose, nutrition, inflammation: What is the relationship? Data from the Dialysis Outcomes and Practices Patterns Study (DOPPS). Nephrol Ther 6: 7-12.

21. Al-Thani H, El-Menyar A, Koshy $\mathrm{V}$, Hussein $A$, Sharaf $A$, et al. (2014) Implications of foot ulceration in haemodialysis patients: A 5-year observational study. J Diab Res 2014: 945075.

22. Hassani K, Aatif T, Alayoud A, Hamzi M, Maoujoud O, et al. (2011) Assessment of nutritional status and prevalence of undernutrition in chronic haemodialysis. Nephrol Ther 7: 310.

23. Elouazzani H, Sirajedine K, Aladib M (2011) Évaluation du statut lipidique chez les hémodialysés chroniques. Nephrol Ther 7: 322-323.

24. Ash S, Campbell K, MacLaughlin H, McCoy E, Chan M, et al. (2006) Evidence based practice guidelines for the nutritional management of chronic kidney disease. Nutr Diet 63: S35-S45.

25. Ndip A, Lavery LA, Boulton AJ (2010) Diabetic foot disease in people with advanced nephropathy and those on renal dialysis. Curr Diab Rep 10: 283-290.

26. Villar E, Zaoui $P$ (2010) Diabetes and chronic kidney disease: Lessons from renal epidemiology. Nephro Ther 6: 585-590.

27. Wolf G, Muller N, Busch M, Eidner G, Kloos C, et al. (2009) Diabetic foot syndrome and renal function in type 1 and 2 diabetes mellitus show close association. Nephrol Dial Transplant 24: 1896-1901.

28. Otte J, van Netten, Woittiez A (2015) The association of chronic kidney disease and dialysis treatment with foot ulceration and major amputation. J Vasc Surg 62: 406-411.

29. Roaas A, Anderson GBJ (1982) Normal range of motion of the hip, knee and ankle joints in male subjects, $30-40$ years of age. Acta Orthop Scand 53: 205-208.

30. Richard JL, Schuldiner S (2008) Epidemiology of diabetic foot problems. Rev Med Interne 29: S222-S230.

31. Cole S (2001) Vascular laboratory practice, part III: lower limb arterial assessment. ( $1^{\text {st }}$ edn), Institute of Physics and Engineering in Medicine, York, UK.

32. Victor A, Michael HC, Pierre A, Matthew AA, Mark AC (2012) Measurement and interpretation of the anklebrachial Index. Circulation 126: 2890-2909.

33. McGill M, Molyneaux L, Yue DK (1998) Use of the Semmes-Weinstein 5.07/10 gram monoflament: The long and the short of it. Diabet Med 15: 615-617.

34. Apelqvist J, Bakker K, van Houtum WH, Schaper NC, International Working Group on the Diabetic Foot (IWGDF) Editorial Board (2008) Practical guidelines on the management and prevention of the diabetic foot: based upon the International Consensus on the Diabetic Foot (2007) Prepared by the International Working Group on the Diabetic Foot. Diabetes Metab Res Rev 24: S181-S187. 